\title{
What the HEC? Clinician Adherence to Evidence-Based Antiemetic Prophylaxis for Highly Emetogenic Chemotherapy
}

Eric J. Roeland, MD; Kathryn J. Ruddy, MD²; Thomas W. LeBlanc, MD³; Ryan D. Nipp, MD; Gary Binder, MBA4; Silvia Sebastiani, $\mathrm{PhD}^{5}$; Ravi Potluri, $\mathrm{PGDM}^{6}$; Luke Schmerold, BS ; Eros Papademetriou, MA';

Lee Schwartzberg, $\mathrm{MD}^{7}$; and Rudolph M. Navari, $\mathrm{MD}^{8}$

\section{ABSTRACT}

\begin{abstract}
Background: Clinician adherence to antiemetic guidelines for preventing chemotherapy-induced nausea and vomiting (CINV) caused by highly emetogenic chemotherapy (HEC) remains poorly characterized. The primary aim of this study was to evaluate individual clinician adherence to HEC antiemetic guidelines. Patients and Methods: A retrospective analysis of patients receiving $\mathrm{HEC}$ was conducted using the IBM Watson Explorys Electronic Health Record Database (2012-2018). HEC antiemetic guideline adherence was defined as prescription of triple prophylaxis (neurokinin-1 receptor antagonist [NK1 RA], serotonin type-3 receptor antagonist, dexamethasone) at initiation of cisplatin or anthracycline + cyclophosphamide (AC). Clinicians who prescribed $\geq 5$ HEC courses were included and individual guideline adherence was assessed, noting the number of prescribing clinicians with $>90 \%$ adherence. Results: A total of 217 clinicians were identified who prescribed 2,543 cisplatin and 1,490 AC courses. Patients $(\mathrm{N}=4,033)$ were primarily women $(63.3 \%)$ and chemotherapy-naive (92\%) with a mean age of 58.6 years. Breast (36\%) and thoracic (19\%) cancers were the most common tumor types. Guideline adherence rates of $>90 \%$ were achieved by $35 \%$ and $58 \%$ of clinicians using cisplatin or AC, respectively. Omission of an NK1 RA was the most common practice of nonadherence. Variation in prophylaxis guideline adherence was considerable for cisplatin (mean, 71\%; SD, 29\%; coefficient of variation [CV], 0.40) and AC (mean, 84\%; SD, 26\%; CV, 0.31 ). Conclusions: Findings showed substantial gaps in clinician adherence to HEC CINV guidelines, including a high variability across clinicians. Clinicians should review their individual clinical practices and ensure adherence to evidence-based CINV guidelines to optimize patient care.
\end{abstract}

J Natl Compr Canc Netw 2020;18(6):676-681 doi: $10.6004 /$ jnccn.2019.7526

\footnotetext{
${ }^{1}$ Massachusetts General Hospital Cancer Center, Boston, Massachusetts; ${ }^{2}$ Mayo Clinic, Rochester Minnesota; ${ }^{3}$ Duke University, Durham, North Carolina; ${ }^{4}$ Helsinn Therapeutics US, Iselin, New Jersey; ${ }^{5}$ Helsinn Therapeutics, Lugano, Switzerland; ${ }^{6}$ SmartAnalyst, New York, New York; ${ }^{7}$ West Cancer Center, Memphis, Tennessee; and ${ }^{8}$ University of Alabama at Birmingham, Birmingham, Alabama.
}

\section{Background}

Despite the availability of evidence-based guidelines for the prevention of chemotherapy-induced nausea and vomiting (CINV), adherence to these guidelines remains suboptimal. In addition to the impact on quality of life (QoL), poorly controlled CINV can lead to chemotherapy treatment delays, dose reductions, and increased healthcare utilization. ${ }^{1}$ Use of prophylactic combination antiemetic regimens targeting multiple biologic mechanisms associated with CINV represent the standard of care for patients with cancer. CINV prophylaxis remains the fundamental strategy, because once patients experience CINV they are 4 times more likely to experience nausea and vomiting in subsequent cycles of chemotherapy. ${ }^{2}$

Several organizations have published antiemetic prophylactic guidelines, including the Multinational Association of Supportive Care in Cancer in collaboration with ESMO (MASCC/ESMO, 2016), ${ }^{3}$ ASCO (2017), ${ }^{4}$ and NCCN (2020). ${ }^{5}$ These evidence-based antiemetic guidelines stratify antiemetic prophylaxis based on the emetic risk of the chemotherapy agents, with highly emetogenic chemotherapy (HEC) being the highest risk category. Cisplatin represents the most widely recognized HEC agent, followed by the combination of anthracycline + cyclophosphamide (AC). For the management of HEC, all guidelines endorse a triple combination regimen of a neurokinin-1 receptor antagonist (NK1 RA), serotonin type-3 RA (5HT3 RA), and dexamethasone (DEX) \pm olanzapine as the evidence-based approach ${ }^{3-5}$; the NCCN Clinical Practice Guidelines in Oncology (NCCN Guidelines) for Antiemesis also recommend another prophylactic olanzapine-based antiemetic combination regimen for HEC. ${ }^{5}$ Data demonstrate that adherence to these guidelines can improve patient-reported outcomes and maintain treatment intensity while decreasing healthcare utilization..$^{1,6,7}$ However, guideline

See page 791 for related article. 
adherence remains poorly integrated into routine clinical practice. ${ }^{1,8-12}$

Moreover, CINV are among the most common toxicities, accounting for most of the avoidable acute care among patients receiving chemotherapy. ${ }^{13}$ In addition, other recent data suggest that rates of avoidable acute care involving CINV are approximately 50\% higher for HEC compared with non-HEC. ${ }^{14}$ Recently, the Centers for Medicare \& Medicaid Services (CMS) instituted the first oncology outcome measure, OP-35. This measure tracks and publicly reports event rates for acute care involving certain toxicities, including CINV, and revises Medicare reimbursement based on observed rates. ${ }^{13}$ Therefore, CINV prophylaxis adherence is critically important because it has implications for patient outcomes and potentially even reimbursement to clinicians and/or health systems.

This study evaluated clinician adherence to HEC prophylactic antiemetic guidelines using current definitions of HEC for patients receiving cisplatin and AC..$^{3-5}$ By enhancing our understanding of individual clinician adherence to CINV guidelines for HEC agents, we hope to highlight current gaps in the clinical uptake of these guidelines and to inform future efforts to ensure patients receive the highest quality evidence-based cancer care.

\section{Patients and Methods}

\section{Data Source and Design}

We performed a retrospective analysis using deidentified patient data from the IBM Watson Explorys Electronic Health Record Database to determine initiation of HEC predominantly in the outpatient setting in October 2012 through August 2018. To exclude patients with low-dose and multiday chemotherapy, we identified patients aged $\geq 18$ years receiving HEC courses with $\geq 7$-day cycles of cisplatin or AC, and any antiemesis prophylaxis medications they received, using National Drug Codes and the Healthcare Common Procedure Coding System. For each patient, we defined a course of chemotherapy as an initial administration followed by periodic subsequent administrations (multiple cycles) that continued until a 90-day treatment gap occurred (maximum of 180 days). We included only patients who were chemotherapy-free $\geq 90$-days prior to and had $\geq 30$ days of data available after initial HEC administration.

We assigned patients to oncologists or hematologists associated with the chemotherapy administered, where such data were available. When multiple clinicians prescribed chemotherapy for a single patient, we assigned the clinician with the most encounters recorded to that patient. We then identified clinicians who prescribed $\geq 5$ courses of cisplatin or $\geq 5$ courses of AC, ranked their guideline adherence, and assessed variation among individual clinicians. We defined antiemetic guideline adherence as triple prophylaxis (ie, NK1 RA + 5HT3 RA + DEX) at the initiation of an HEC course and focused on the number of clinicians who achieved $>90 \%$ adherence.

\section{Statistical Analysis}

We analyzed patient characteristics, HEC courses, and clinician practice patterns using descriptive statistics. Clinician HEC CINV prophylaxis adherence was categorized into deciles for each HEC, noting the clinicians with $\leq 90 \%$ adherence. Variability in individual clinician adherence to guidelines was assessed by evaluating the proportion of clinicians within each of the 10 deciles of adherence, and by calculating the coefficient of variation (CV) indicating the degree of dispersion around the mean. ${ }^{15}$ We also evaluated interclinician variability by comparing the adherence rates for the least adherent $20 \%$ of clinicians versus the most adherent $20 \%$ and calculating a ratio, consistent with literature. ${ }^{16}$ All study analyses were conducted using SAS 9.4 (SAS Institute Inc.) and Microsoft Excel (Microsoft).

\section{Results}

We identified 8,176 courses of cisplatin and AC representing $51 \%$ of HEC courses, of which 6,081 were assigned to 1,265 clinicians. Excluding courses assigned to clinicians who treated $<5$ patients with the selected chemotherapies resulted in 2,543 cisplatin and 1,490 AC courses (Table 1). These courses were assigned to 217 clinicians who averaged 19 courses of therapy $(>90 \%$ given to patients with no prior chemotherapy). Mean age for patients receiving HEC was 58.6 years. Most patients were women $(63 \%)$ and from the Midwest $(73 \%)$. Breast cancer was the most common tumor type treated using these HEC regimens (36\%) given the inclusion of AC.

Guideline adherence rates of $>90 \%$ were achieved by $35 \%$ and $58 \%$ of clinicians using cisplatin or AC, respectively. Clinicians with $\leq 90 \%$ adherence did not provide guideline-recommended antiemetics with an average of $>40 \%$ of cisplatin and $>35 \%$ of AC initiations; these clinicians were distributed over a broad range of lower adherence rates (Figure 1, Table 2). Variation in CINV prophylaxis guideline adherence was considerable for cisplatin (mean, $71 \%$; SD 29\%; $\mathrm{CV}, 0.40$ ) and $\mathrm{AC}$ (mean, $84 \%$; SD, $26 \%$; CV, 0.31). When clinicians were ranked in ascending order by adherence rates for cisplatin, the 20th percentile of clinicians was $43 \%$ adherent, whereas the 80 th percentile was $100 \%$ adherent, a ratio of 2.3 times more adherent to CINV prophylaxis guidelines. For AC, the respective adherence rates were $70 \%$ and $100 \%$, resulting in a ratio of 1.4 times greater adherence among the top quintile compared with the lowest $20 \%$ of clinicians. 


\section{Table 1. HEC and Patient Characteristics}

\begin{tabular}{|lc|}
\hline Characteristic & $\mathbf{n}$ \\
\hline Patients & 4,033 \\
\hline Mean age, y & 58.6 \\
\hline Women & $2,552(63.3 \%)$ \\
\hline Cancer type & \\
\hline Breast & $1,452(36 \%)$ \\
\hline Thoracic & $766(19 \%)$ \\
\hline Head and neck & $524(13 \%)$ \\
\hline Gastrointestinal & $202(5 \%)$ \\
\hline Other & $1,089(27 \%)$ \\
\hline Total & $4,033(100 \%)$ \\
\hline HEC courses & 4,033 \\
\hline Type of HEC & \\
\hline Cisplatin & $2,543(63.1 \%)$ \\
\hline AC & $1,490(36.9 \%)$ \\
\hline Median number of cycles/patient & 4 \\
\hline Clinicians using $\geq 5$ prescribed cisplatin or AC courses & \\
\hline Physicians & $189(87.1 \%)$ \\
\hline Advanced practice providers & $28(12.9 \%)$ \\
\hline Total & 217 \\
\hline US region of treatment & $543(13.5 \%)$ \\
\hline Midwest & $503(12.5 \%)$ \\
\hline South & $50(1.2 \%)$ \\
\hline Nest & \\
\hline
\end{tabular}

Abbreviations: AC, anthracycline + cyclophosphamide; HEC, highly emetogenic chemotherapy.

aThe following ICD-9 and ICD-10 codes were used to identify each cancer type: breast cancer: 174. $\times$, 175. $\times$, C50.X; thoracic (respiratory): $160 . \times-165 . \times$, C30-C39; head and neck (lip, oral cavity, and pharynx): $140 . \times-149 . \times$, C00-C15; gastrointestinal (digestive): 150.×-159.×, C15-C26, C45.1, C48.×.

Across cisplatin and AC, omission of NK1 RA was the principal cause of nonadherence in $>90 \%$ of cases. Olanzapine was unlikely a driver of NK1 RA omission given its low use, with a total of 116 prescriptions (3\% of courses) identified during the study period.

\section{Discussion}

We investigated individual clinicians' rates of adherence to CINV guidelines for HEC and found that a considerable proportion of clinicians did not achieve $>90 \%$ adherence to guideline-concordant triple antiemetic prophylaxis for HEC. A novel finding of this study was the magnitude of variability of adherence across clinicians. Notably, most clinicians prescribing cisplatin had considerably lower adherence and widely varying CINV prophylaxis patterns compared with those prescribing AC. This may be related to clinician experience in treating patients with breast cancer, who are at

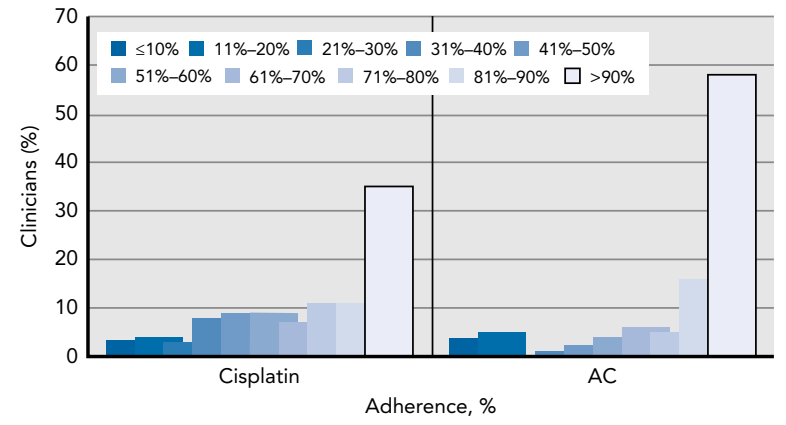

Figure 1. Individual clinician adherence to antiemetic prophylaxis guidelines for highly emetogenic chemotherapy (data from 2012-2018).

Abbreviation: AC, anthracycline + cyclophosphamide.

higher risk of CINV based on individual risks factors, such as sex, age, and low alcohol use. ${ }^{17,18}$ Strong evidence supports the use of effective CINV prophylaxis, as detailed by multiple guidelines, ${ }^{3-5}$ and therefore the suboptimal adherence we found demonstrates a compelling need to improve CINV guideline adherence. ${ }^{10}$

Adherence to available CINV prophylaxis guidelines has the potential to improve patient outcomes. For example, in a prospective, multicenter observational study among chemotherapy-naïve patients, Aapro et $\mathrm{al}^{6} \mathrm{re}^{\mathrm{r}}$ ported reduced incidence of CINV over a 5-day period after implementing guideline-concordant antiemetic prophylaxis. Similarly, another prospective observational study in 1,300 patients showed that adherence to antiemetic prophylaxis guidelines resulted in lower

Table 2. Individual Clinician Adherence Levels

\begin{tabular}{|lrr|}
\hline & \multicolumn{2}{c|}{$\begin{array}{c}\text { Clinicians at Each } \\
\text { Adherence Level (\%) }\end{array}$} \\
\cline { 2 - 3 } & Cisplatin & AC \\
\hline Adherence with antiemetic guidelines & 3 & 4 \\
\hline$\leq 10 \%$ & 4 & 5 \\
\hline $11 \%-20 \%$ & 3 & 0 \\
\hline $21 \%-30 \%$ & 8 & 1 \\
\hline $31 \%-40 \%$ & 9 & 2 \\
\hline $41 \%-50 \%$ & 9 & 4 \\
\hline $51 \%-60 \%$ & 7 & 6 \\
\hline $61 \%-70 \%$ & 11 & 5 \\
\hline $71 \%-80 \%$ & 11 & 16 \\
\hline $81 \%-90 \%$ & 35 & 58 \\
\hline$>90 \%$ & 2,543 & 1,490 \\
\hline Patients, $n$ & 176 & 111 \\
\hline Clinicians, $n$ & 71 & 84 \\
\hline Mean adherence, \% & 3 & \\
\hline
\end{tabular}

Abbreviation: AC, anthracycline + cyclophosphamide. 
incidence of CINV. ${ }^{19}$ Guideline recommendations are informed by evidence that poorly controlled CINV directly affects patient care and healthcare utilization. A meta-analysis showed that CINV resulted in poor health-related QoL, ${ }^{20}$ and poorly controlled CINV accounted for a large proportion of patients experiencing decreased QoL in a prospective analysis. ${ }^{21}$ In addition to the impact on QoL, CINV causes psychologic distress, impairs nutrition, and disrupts treatment delivery. ${ }^{22}$ Thus, reevaluating and maximizing clinician adherence to evidence-based CINV prophylaxis guidelines may improve patient outcomes.

Despite recognition from ASCO that the development of effective antiemetic therapies represents one of the top 5 advances in oncology over the past 50 years, ${ }^{23}$ our findings align with prior work suggesting that CINV remains a significant challenge in oncology. ${ }^{9-12}$ A 2016 national survey of 5,422 patients with lung or colorectal cancer found that nearly $30 \%$ wanted additional help in controlling their nausea and vomiting, with $68 \%$ reporting moderate to severe symptoms. ${ }^{24}$ In fact, among 1.5 million emergency department visits from 2006 through 2015, nausea and vomiting was the fifth most common treatment-related complication, with $67 \%$ of visits resulting in inpatient admission. ${ }^{25}$ In addition, Basch et $\mathrm{al}^{26}$ found nausea and emesis to be among the chemotherapy symptoms for which systematic monitoring and reporting of toxicities led to better QoL, lower emergency department use, and greater survival. Collectively, these data suggest that CINV remains a significant clinical challenge.

Furthermore, poorly controlled CINV can directly impact healthcare utilization and cost. ${ }^{27}$ Several studies have evaluated the economic impact of CINV on healthcare costs in the United States. Projected additional costs per patient for poorly controlled CINV range from $>\$ 7,631$ to $\$ 10,699$ during the first 5 days after chemotherapy administration, including outpatient visits, emergency department visits, and hospitalizations (adjusted to 2019 \$USD). ${ }^{21,28}$ Reflecting the prevalence and cost of this toxicity, CMS has recognized CINV as 1 of 10 potentially avoidable causes of acute care postchemotherapy that are now part of the OP-35 measure. CMS plans to publicize hospital outpatient rates for these events and reduce Medicare reimbursement for institutions below national averages. ${ }^{13}$

Oncologists strive to provide the best care for patients and minimize treatment-related adverse effects, including CINV, and patients expect their clinicians to follow guidelines that minimize toxicity and maximize QoL. Consequently, our findings highlight the need to better understand existing barriers that continue to lead to low adherence rates to CINV guidelines. Notably, clinicians and nurses markedly underestimate the incidence of delayed CINV compared with patient experience. ${ }^{9,29,30}$ Among potential explanations, patients may not inform their oncologists of their poorly controlled CINV due to their desire to focus clinical discussions on tumor response and treatment options, as well as misperceptions that nausea and vomiting indicate that the chemotherapy is effective. Additionally, patients may not want to report their poorly controlled symptoms for fear oncologists may reduce or delay their cancer treatment. Our findings highlight the need for efforts to improve adherence to antiemetic guidelines. In particular, we found a subset of prescribing clinicians who did achieve $>90 \%$ adherence to CINV guidelines, suggesting such adherence to evidence-based guidelines is feasible and underscoring opportunities for future research to learn from these clinicians.

Strengths of this study include that the large, population-based design focused on 2 established HEC regimens. However, we note several limitations of this study. First, we lacked institution-specific data, which limited our ability to distinguish between individual clinician preference versus institution-mandated or default practices. We also were not able to analyze any clinicianspecific variables that may be associated with adherence. Second, our selected database had limited geographic representation, with an overrepresentation of the Midwest. Third, we acknowledge that the causes for lack of adherence to the CINV guidelines are multifactorial, and we lacked information about important factors such as clinician knowledge of CINV guidelines, their perceptions of CINV, and institution-based practice patterns. ${ }^{9,30}$ This is compounded by data limitations that precluded determination of different clinical sites of care, such as academic versus community settings. Fourth, we were unable to determine causality and explore specific barriers to CINV guideline adherence given our retrospective study design. Fifth, we did not capture any information regarding costs associated with CINV guideline adherence, including emergency department visits, hospitalizations, and unplanned hydrations. Sixth, we limited our evaluation to the use of NK1 RA, 5HT3 RA, and DEX given the few prescriptions for olanzapine ${ }^{31}$ and lack of association with NK1 RA omission. Finally, our evaluations excluded recent CINV prophylaxis for carboplatin, because its recategorization from moderately emetogenic chemotherapy to HEC occurred near the end of the data period.

Future research should evaluate the barriers to CINV guideline adherence. Potential barriers may include the lack of incorporated evidence-based antiemetic guidelines into the electronic medical record, delayed updates to supportive care medications given slow-moving institutional timelines, limitations in clinician understanding of recent guidelines updates, and/ or preferred clinical practice. Additionally, supportive 
oncology medications may not be a priority for prescribing oncology clinicians given the challenges of staying up to date with cancer-directed therapies, particularly if combined with a simple lack of awareness of antiemetic guidelines, in addition to lack of measurement and management.

\section{Conclusions}

Substantial gaps exist in clinician adherence to HEC CINV prophylaxis guidelines. Upfront triple antiemetic prophylaxis in the HEC setting remains low and clinician adherence to guidelines is highly variable. With substantial evidence demonstrating the benefits of guideline adherence and new CMS measures tracking toxicityrelated hospitalizations, efforts to improve adherence to CINV prophylaxis are needed to ensure clinicians and health systems implement changes to meet patient needs and reduce avoidable acute care.

Submitted August 4, 2019; accepted for publication December 19, 2019.

Author contributions: Study design: Roeland, Binder, Schmerold, Navari. Data collection: Potluri, Papdemetriou. Data analysis: Roeland, Ruddy, LeBlanc,
Nipp, Binder, Sebastiani, Schmerold, Schwartzberg, Navari. Drafting of manuscript: Roeland, Ruddy, LeBlanc, Nipp, Binder, Sebastiani, Schmerold, Schwartzberg, Navari. Critical revision: All authors.

Disclosures: Dr. Roeland has disclosed that he has received consulting fees from Asahi Kasei Pharmaceuticals, DRG Consulting. American Imaging Management, Napo Pharmaceuticals, Immuneering Corporation, and Prime Oncology; and was a scientific advisor for Heron Pharmaceuticals, Vector Oncology, Oragenics, Inc., Galera Pharmaceuticals, and Enzychem Lifesciences Pharmaceutical Company. Dr. LeBlanc has disclosed that he has received grant/research support from Jazz Pharmaceuticals and Seattle Genetics; has served as a scientific advisor for AbbVie, Agios, Amgen, Daiichi-Sankyo, Heron Therapeutics, Medtronic, and Otsuka; receives royalties from UpToDate; and receives consultant fees from Agios, AstraZeneca, CareVive, Flatiron, Helsinn Therapeutics, Otsuka, Pfizer, Seattle Genetics, and Welvie. Mr. Binder and Dr. Sebastiani have disclosed that they are employees of Helsinn Therapeutics and Helsinn Healthcare. Mr. Potluri, Mr. Schmerold, and Mr. Papdemetriou have disclosed that they have received consulting fees from Helsinn Therapeutics. Dr. Schwartzberg has disclosed that he receives consulting fees from or is a scientific advisor for Amgen, Pfizer, Helsinn Therapeutics Genentech/Roche, Genomic Health, Bristol-Myers Squibb, Myriad, AstraZeneca, Bayer, Spectrum, and Napo. The remaining authors have disclosed that they have no financial interests, arrangements, affiliations, or commercial interests with the manufacturers of any products discussed in this article or their competitors.

Funding: This study was sponsored by Helsinn Therapeutics (US), Inc. Dr. Roeland is also sponsored by the Cambia Health Foundation Sojourns Scholar Award.

Correspondence: Eric J. Roeland, MD, Massachusetts General Hospital Cancer Center, 55 Fruit Street, Yawkey 7E, Boston, MA 02114. Email: eroeland@mgh.harvard.edu

\section{References}

1. Schwartzberg L, Harrow B, Lal LS, et al. Resource utilization for chemotherapy-induced nausea and vomiting events in patients with solid tumors treated with antiemetic regimens. Am Health Drug Benefits 2015; 8:273-282.

2. Schwartzberg L, Szabo S, Gilmore J, et al. Likelihood of a subsequent chemotherapy-induced nausea and vomiting (CINV) event in patients receiving low, moderately or highly emetogenic chemotherapy (LEC/MEC/HEC). Curr Med Res Opin 2011:27:837-845.

3. Herrstedt J, Roila F, Warr D, et al. 2016 Updated MASCC/ESMO consensus recommendations: prevention of nausea and vomiting following high emetic risk chemotherapy. Support Care Cancer 2017;25:277-288.

4. Hesketh PJ, Kris MG, Basch E, et al. Antiemetics: American Society of Clinical Oncology clinical practice guideline update. J Clin Oncol 2017 35:3240-3261.

5. Ettinger DS, Berger MJ, Barbour S, et al. NCCN Clinical Practice Guidelines in Oncology: Antiemesis. Version 1.2020. Accessed April 14, 2020. To view the most recent version, visit NCCN.org

6. Aapro M, Molassiotis A, Dicato M, et al. The effect of guideline-consistent antiemetic therapy on chemotherapy-induced nausea and vomiting (CINV): the Pan European Emesis Registry (PEER). Ann Oncol 2012;23. 1986-1992.

7. Dranitsaris G, Mazzarello S, Smith S, et al. Measuring the impact of guideline-based antiemetic therapy on nausea and vomiting control in breast cancer patients with multiple risk factors. Support Care Cancer 2015;24:1563-1569.

8. Van Laar ES, Desai JM, Jatoi A. Professional educational needs for chemotherapy-induced nausea and vomiting (CINV): multinational survey results from 2388 health care providers. Support Care Cancer 2015;23: 151-157.

9. Clark-Snow R, Affronti ML, Rittenberg CN. Chemotherapy-induced nausea and vomiting (CINV) and adherence to antiemetic guidelines: results of a survey of oncology nurses. Support Care Cancer 2018;26:557-564

10. Mahendraratnam N, Farley JF, Basch E, et al. Characterizing and assessing antiemetic underuse in patients initiating highly emetogenic chemotherapy. Support Care Cancer 2019;27:4525-4534.

11. O'Sullivan CC, Van Houten HK, Sangaralingham LR, et al. Ten-year trends in antiemetic prescribing in patients receiving highly emetogenic chemotherapy. J Natl Compr Canc Netw 2018;16:294-299.

12. Roeland E, Ruddy KJ, LeBlanc TW, et al. Gaps in compliance with current antiemetic guidelines for highly emetogenic chemotherapy [abstract].
Presented at the NCCN 23rd Annual Conference; March 22-23, 2018; Orlando Florida. Abstract AB2018-36.

13. Centers for Medicare \& Medicaid Services. CMS Finalizes Hospital Outpatient Prospective Payment Changes for 2017. Accessed June 9, 2019. Available at: https://www.cms.gov/newsroom/fact-sheets/ cms-finalizes-hospital-outpatient-prospective-payment-changes-2017

14. Roeland EJ, LeBlanc TW, Ruddy KJ, et al. Avoidable acute care use associated with nausea and vomiting among patients receiving highly emetogenic chemotherapy or oxaliplatin [abstract]. Presented at the NCCN 2019 Annual Conference; March 21-23, 2019; Orlando, Florida. Abstract BPI19-019.

15. O'Sullivan JW, Stevens S, Oke J, et al. Practice variation in the use of tests in UK primary care: a retrospective analysis of 16 million tests performed over 3.3 million patient years in 2015/16. BMC Med 2018;16:229.

16. Mays GP, Smith SA. Geographic variation in public health spending: correlates and consequences. Health Serv Res 2009;44:1796-1817.

17. Molassiotis A, Aapro M, Dicato M, et al. Evaluation of risk factors predicting chemotherapy-related nausea and vomiting: results from a European prospective observational study. J Pain Symptom Manage 2014; 47:839-848.

18. Dranitsaris G, Molassiotis A, Clemons M, et al. The development of a prediction tool to identify cancer patients at high risk for chemotherapyinduced nausea and vomiting. Ann Oncol 2017;28:1260-1267.

19. Gilmore JW, Peacock NW, Gu A, et al. Antiemetic guideline consistency and incidence of chemotherapy-induced nausea and vomiting in US community oncology practice: INSPIRE Study. J Oncol Pract 2014;10: 68-74.

20. Sommariva S, Pongiglione B, Tarricone R. Impact of chemotherapyinduced nausea and vomiting on health-related quality of life and resource utilization: a systematic review. Crit Rev Oncol Hematol 2016;99: 13-36.

21. Haiderali $A$, Menditto $L$, Good $M$, et al. Impact on daily functioning and indirect/direct costs associated with chemotherapy-induced nausea and vomiting (CINV) in a U.S. population. Support Care Cancer 2011;19: 843-851.

22. Farrell C, Brearley SG, Pilling M, et al. The impact of chemotherapyrelated nausea on patients' nutritional status, psychological distress and quality of life. Support Care Cancer 2013;21:59-66.

23. Center to Advance Palliative Care. National Palliative Care Registry. 2015 Accessed January 2, 2020. Available at: registry.capc.org 
24. Walling $\mathrm{AM}$, Keating $\mathrm{NL}$, Kahn $\mathrm{KL}$, et al. Lower patient ratings of physician communication are associated with unmet need for symptom management in patients with lung and colorectal cancer. J Oncol Pract 2016;12:e654-669.

25. Jairam V, Lee V, Park HS, et al. Treatment-related complications of systemic therapy and radiotherapy. JAMA Oncol 2019;5: 1028-1035.

26. Basch $E$, Deal AM, Dueck $A C$, et al. Overall survival results of a trial assessing patient-reported outcomes for symptom monitoring during routine cancer treatment. JAMA 2017;318:197-198.

27. Bloechl-Daum B, Deuson RR, Mavros P, et al. Delayed nausea and vomiting continue to reduce patients' quality of life after highly and moderately emetogenic chemotherapy despite antiemetic treatment. J Clin Oncol 2006:24:4472-4478.
28. Burke TA, Wisniewski T, Ernst FR. Resource utilization and costs associated with chemotherapy-induced nausea and vomiting (CINV) following highly or moderately emetogenic chemotherapy administered in the US outpatient hospital setting. Support Care Cancer 2011;19:131-140.

29. Majem M, Moreno ME, Calvo N, et al. Perception of healthcare providers versus patient reported incidence of chemotherapy-induced nausea and vomiting after the addition of NK-1 receptor antagonists. Support Care Cancer 2011;19:1983-1990.

30. Grunberg SM, Deuson RR, Mavros P, et al. Incidence of chemotherapyinduced nausea and emesis after modern antiemetics. Cancer 2004;100: 2261-2268.

31. Navari RM, Qin R, Ruddy KJ, et al. Olanzapine for the prevention of chemotherapy-induced nausea and vomiting. N Engl J Med 2016;375: 134-142.

\section{Questions About Cancer?
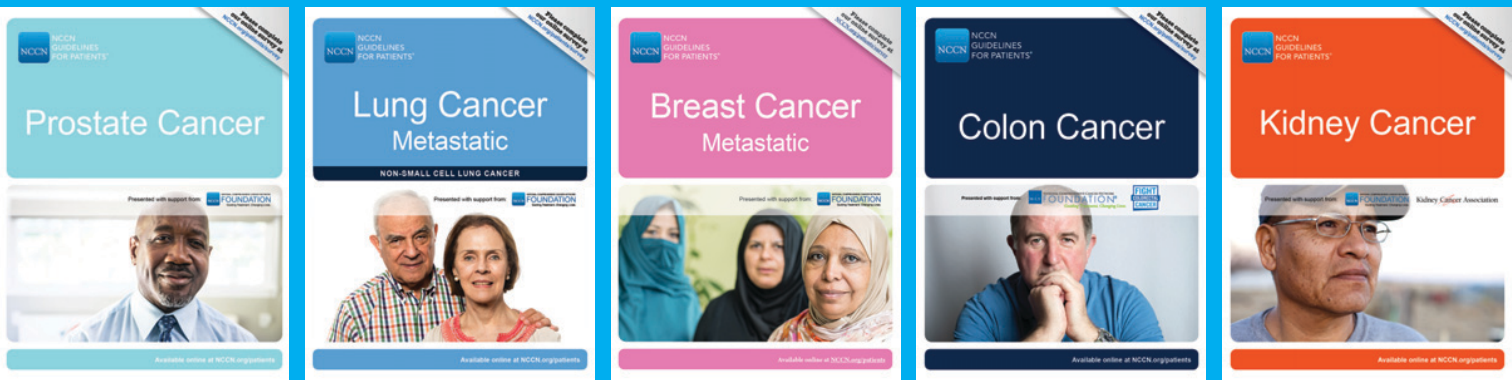

\section{NGGN Cuidelines for Patients ${ }^{\circledR}$ provide expert cancer treatment information.}

